\title{
A note on the distance-balanced property of generalized Petersen graphs*
}

\author{
Rui Yang, Xinmin Hou, ${ }^{\dagger}$ Ning Li, Wei Zhong \\ Department of Mathematics \\ University of Science and Technology of China \\ Hefei, Anhui, 230026, P. R. China
}

Submitted: Aug 25, 2008; Accepted: Nov 13, 2009; Published: Nov 24, 2009

Mathematics Subject Classifications: 05C75, 05C12

\begin{abstract}
A graph $G$ is said to be distance-balanced if for any edge $u v$ of $G$, the number of vertices closer to $u$ than to $v$ is equal to the number of vertices closer to $v$ than to $u$. Let $G P(n, k)$ be a generalized Petersen graph. Jerebic, Klavžar, and Rall [Distance-balanced graphs, Ann. Comb. 12 (2008) 71-79] conjectured that: For any integer $k \geqslant 2$, there exists a positive integer $n_{0}$ such that the $\operatorname{GP}(n, k)$ is not distance-balanced for every integer $n \geqslant n_{0}$. In this note, we give a proof of this conjecture.
\end{abstract}

Keywords: generalized Petersen graph, distance-balanced graph

\section{Introduction}

Let $G$ be a simple undirected graph and $V(G)(E(G))$ be its vertex (edge) set. The distance $d(u, v)$ between vertices $u$ and $v$ of $G$ is the length of a shortest path between $u$ and $v$ in $G$. For a pair of adjacent vertices $u, v \in V(G)$, let $W_{u v}$ denote the set of all vertices of $G$ closer to $u$ than to $v$, that is

$$
W_{u v}=\{x \in V(G) \mid d(u, x)<d(v, x)\} .
$$

Similarly, let ${ }_{u} W_{v}$ be the set of all vertices of $G$ that are at the same distance to $u$ and $v$, that is

$$
{ }_{u} W_{v}=\{x \in V(G) \mid d(u, x)=d(v, x)\} .
$$

A graph $G$ is called distance-balanced if

$$
\left|W_{u v}\right|=\left|W_{v u}\right|
$$

${ }^{*}$ The work was supported by NNSF of China (No.10701068).

${ }^{\dagger}$ Corresponding author: xmhou@ustc.edu.cn 
holds for every pair of adjacent vertices $u, v \in V(G)$.

Let $u v$ be an arbitrary edge of $G$. Then $d(u, x)-d(v, x) \in\{1,0,-1\}$. Hence $W_{u v}=$ $\{x \in V(G) \mid d(v, x)-d(u, x)=1\},{ }_{u} W_{v}=\{x \in V(G) \mid d(v, x)-d(u, x)=0\}$, and $W_{v u}=\{x \in V(G) \mid d(v, x)-d(u, x)=-1\}$ form a partition of $V(G)$. The following proposition follows immediately from the above comments.

Proposition 1 If $\left|W_{u v}\right|>|V(G)| / 2$ for an edge uv of $G$, then $G$ is not distance-balanced.

Let $n \geqslant 3$ be a positive integer, and let $k \in\{1, \ldots, n-1\} \backslash\{n / 2\}$. The generalized Petersen graph $G P(n, k)$ is defined to have the following vertex set and edge set:

$$
\begin{gathered}
V(G P(n, k))=\left\{u_{i} \mid i \in \mathbb{Z}_{n}\right\} \cup\left\{v_{i} \mid i \in \mathbb{Z}_{n}\right\}, \\
E(G P(n, k))=\left\{u_{i} u_{i+1} \mid i \in \mathbb{Z}_{n}\right\} \cup\left\{v_{i} v_{i+k} \mid i \in \mathbb{Z}_{n}\right\} \cup\left\{u_{i} v_{i} \mid i \in \mathbb{Z}_{n}\right\} .
\end{gathered}
$$

Jerebic, Klavžar, Rall [1] posed the following conjecture.

Conjecture 1 For any integer $k \geqslant 2$, there exists a positive integer $n_{0}$ such that the generalized Petersen graph $G P(n, k)$ is not distance-balanced for every integer $n \geqslant n_{0}$.

Motivated by this conjecture, Kutnar et al. [3] studied the strongly distance-balanced property of the generalized Petersen graphs and gave a slightly weaker result that: For any integer $k \geqslant 2$ and $n \geqslant k^{2}+4 k+1$, the generalized Petersen graph $\operatorname{GP}(n, k)$ is not strongly distance-balanced (strongly distance-balanced graph was introduced by Kutnar et al. in $[2])$.

In this note, we prove the following theorem.

Theorem 2 For any integer $k \geqslant 2$ and $n>6 k^{2}, G P(n, k)$ is not distance-balanced.

Theorem 2 gives a positive answer to Conjecture 1 .

\section{The Proof of Theorem 2}

First we give a direct observation.

Proposition 3 For any $i=0,1,2, \ldots, n-1, d\left(u_{0}, u_{i}\right)-d\left(v_{0}, u_{i}\right)=1$ if and only if there exists a shortest path from $u_{0}$ to $u_{i}$ which passes through the edge $u_{0} v_{0}$ first.

We call the cycle induced by the vertices $\left\{u_{0}, u_{1}, \cdots, u_{n-1}\right\}$ the outer cycle of $G P(n, k)$, and the cycles induced by the vertices $\left\{v_{0}, v_{1}, \cdots, v_{n-1}\right\}$ the inner cycles of $\operatorname{GP}(n, k)$. The edge $u_{i} v_{i}(0 \leqslant i \leqslant n-1)$ is called a spoke of $G P(n, k)$.

Proposition 4 Let $G P(n, k)$ be a generalized Petersen graph with $n \geqslant 6 k$ and $k \geqslant 2$. If $3 k \leqslant i \leqslant n-3 k$, then there exists a shortest path between $u_{0}$ and $u_{i}$ which passes through the edge $u_{0} v_{0}$ first. 
Proof. By symmetry, we only need consider the case $3 k \leqslant i \leqslant n / 2$. Let $P\left(u_{0}, u_{i}\right)$ be a shortest path between $u_{0}$ and $u_{i}$. Note that the path between $u_{0}$ and $u_{i}$ contained in the outer cycle has length $i$. The path:

$$
u_{0} \rightarrow v_{0} \rightarrow v_{k} \rightarrow v_{2 k} \rightarrow v_{3 k} \rightarrow u_{3 k} \rightarrow u_{3 k+1} \rightarrow \cdots \rightarrow u_{i}
$$

between $u_{0}$ and $u_{i}$ has length $5+i-3 k$. Since $k \geqslant 2, i+5-3 k<i$. Hence $P\left(u_{0}, u_{i}\right)$ contains spokes. Let $u_{s} v_{s}$ and $v_{l} u_{l}$ be the first spoke and the last one in $P\left(u_{0}, u_{i}\right)$, respectively. If $s=0$, then the result follows. If $s>0$, let $P\left(u_{s}, u_{l}\right)$ be the segment of $P\left(u_{0}, u_{i}\right)$ from $u_{s}$ to $u_{l}$. Define a map $f: V\left(P\left(u_{s}, u_{l}\right)\right) \mapsto V(G P(n, k))$ such that $f\left(u_{j}\right)=u_{j-s}$ and $f\left(v_{j}\right)=v_{j-s}$ for $u_{j} \in V\left(P\left(u_{s}, u_{l}\right)\right)$. Then the segment $f\left(P\left(u_{s}, u_{l}\right)\right)$ is a segment from $u_{0}$ to $u_{l-s}$ which first passes through the edge $u_{0} v_{0}$. Hence the path which first passes through the segment $P\left(u_{0}, u_{l-s}\right)$, then from $u_{l-s}$ to $u_{i}$ along the outer cycle is a shortest path between $u_{0}$ and $u_{i}$, as desired.

In what follows, we give the proof of the main theorem.

Proof of Theorem 2: By Proposition 4, there exists a shortest path from $u_{0}$ to $u_{i}$ which passes through $u_{0} v_{0}$ first for each $3 k \leqslant i \leqslant n-3 k$. By Proposition $3, d\left(u_{0}, u_{i}\right)-$ $d\left(v_{0}, u_{i}\right)=1$. Hence there are more than $n-6 k$ vertices in the outer cycle which satisfy $d\left(u_{0}, u_{i}\right)-d\left(v_{0}, u_{i}\right)=1$.

Now we count the number of vertices in the inner cycle of $G P(n, k)$ satisfying $d\left(u_{0}, v_{i}\right)$ $d\left(v_{0}, v_{i}\right)=1$. For $i=m k(m=0,1,2, \cdots,\lfloor n / 2 k\rfloor)$, it is easy to check that $d\left(u_{0}, v_{i}\right)=m+$ 1 and $d\left(v_{0}, v_{i}\right)=m$. Hence $d\left(u_{0}, v_{i}\right)-d\left(v_{0}, v_{i}\right)=1$. By symmetry, $d\left(u_{0}, v_{i}\right)-d\left(v_{0}, v_{i}\right)=1$ for $i=n-m k(m=1,2, \cdots,\lfloor n / 2 k\rfloor)$. Hence there are at least $2\lfloor n / 2 k\rfloor$ vertices in the inner cycle satisfying $d\left(u_{0}, v_{i}\right)-d\left(v_{0}, v_{i}\right)=1$.

If $n \geqslant 6 k^{2}$, then the number of the vertices $x$ satisfying $d\left(u_{0}, x\right)-d\left(v_{0}, x\right)=1$ is more than $n-6 k+2\lfloor n / 2 k\rfloor \geqslant n-6 k+2\left\lfloor 6 k^{2} / 2 k\right\rfloor=n$. Hence $\left|W_{v_{0} u_{0}}\right|>n=|V(G P(n, k))| / 2$. By Proposition 1, GP(n,k) is not distance-balanced for $n \geqslant 6 k^{2}$ and $k \geqslant 2$.

\section{References}

[1] J. Jerebic, S. Klavžar, D. F. Rall, Distance-balanced graphs, Ann.Comb. 12 (2008) 71-79.

[2] K. Kutnar, A. Malnič, D. Marušič, Š. Miklavič, Distance-balanced graphs: symmetry conditions, Discrete Math. 306 (2006), 1881-1894.

[3] K. Kutnar, A. Malnič, D. Marušič, S. Miklavič, The strongly distance-balanced property of the generalized Petersen graphs, Ars math Contemp., 2 (2009), 41-47. 